\title{
Les tumeurs neuroendocrines
}

\section{Neuroendrocine Tumours}

\section{Rohmer (Ancien président du GTE)}

(C) Lavoisier SAS 2019

Les tumeurs neuroendocrines (TNE) sont issues de cellules dérivées du système endocrinien diffus. Les plus communes sont les tumeurs carcinoïdes ayant pour origine les poumons, l'intestin grêle, l'appendice, le rectum ou le thymus, ainsi que les tumeurs endocrines pancréatiques. Elles comprennent aussi les TNE des parathyroïdes, de l'hypophyse, les carcinomes médullaires de la thyroïde, les phéochromocytomes et les paragangliomes (PGL). Elles peuvent être fonctionnelles avec des symptômes consécutifs à des sécrétions hormonales ou non fonctionnelles. Elles sont le plus souvent sporadiques, mais peuvent aussi s'intégrer dans des syndromes héréditaires (néoplasies endocriniennes multiples 1 et 2 , etc.).

L'incidence des TNE digestives est d'environ 5/100 000, mais leur prévalence en France est d'environ 20 cas pour 100000 habitants, ce qui s'explique par une durée de vie longue dans les cas de TNE bien différenciées.

Les TNE digestives sont encore bien trop ignorées par le monde médical, et leur prise en charge nécessite obligatoirement l'avis multidisciplinaire d'équipes de référence des réseaux RENATEN et TENPATH, eux-mêmes issus du Groupe français d'étude des tumeurs neuroendocrines
(GTE). Ce numéro d'Oncologie permet d'évoquer les principaux outils diagnostiques et thérapeutiques de la prise en charge des TNE, en particulier digestives, pulmonaires, ainsi que le diagnostic des PGL et des phéochromocytomes.

En amont des discussions en réunion de concertation pluridisciplinaire (RCP) RENATEN, il est essentiel de disposer d'une excellente et rigoureuse analyse histologique de la tumeur et/ou de ses métastases. Le caractère différencié ou non de la TNE doit être précisé, de même que le grade en fonction de l'index mitotique ou du Ki67, qui sont des indices de prolifération cellulaire. Il est également essentiel de déterminer le stade TNM dès que possible.

La place et l'utilité des biomarqueurs, tels la chromogranine A ou certains dosages hormonaux, les compléments diagnostiques et pronostiques de la biologie moléculaire, de la génétique, l'imagerie standard ou celle reposant sur la présence à la surface des cellules tumorales des récepteurs à la somatostatine, seront abordées dans les chapitres de ce numéro spécial. Enfin, la chronologie des traitements qu'ils soient classiques ou innovants a également beaucoup évolué grâce à l'établissement de recommandations précises par les sociétés savantes.

V. Rohmer $(\varangle)$

Université d'Angers, F-49035 Angers, France

e-mail : ViRohmer@chu-angers.fr 